\title{
Collectif, La chanson de geste et le sacré
}

\section{Gauthier Grüber}

\section{(2) OpenEdition}

\section{Journals}

Édition électronique

URL : https://journals.openedition.org/ccm/7977

DOI : $10.4000 / \mathrm{ccm} .7977$

ISSN : 2119-1026

\section{Éditeur}

Centre d'études supérieures de civilisation médiévale/Université de Poitiers

\section{Édition imprimée}

Date de publication : 1 septembre 2021

Pagination : 252-256

ISBN : 978-2-490783-10-6

ISSN : 0007-9731

\section{Référence électronique}

Gauthier Grüber, "Collectif, La chanson de geste et le sacré », Cahiers de civilisation médiévale [En ligne] 255 | 2021, mis en ligne le 01 septembre 2021, consulté le 04 février 2022. URL : http:// journals.openedition.org/ccm/7977 ; DOI : https://doi.org/10.4000/ccm.7977

La revue Cahiers de civilisation médiévale est mise à disposition selon les termes de la Licence Creative Commons Attribution - Pas d'Utilisation Commerciale - Pas de Modification 4.0 International. 
La chanson de geste et le sacré, actes du $\mathrm{X}^{\mathrm{e}}$ colloque international de la section française de la Société Rencesvals (Clermont-Ferrand, 18-20 octobre 2017), Nathalie Bragantini-MaILlard, Émilie GoudEAU, Françoise LAurent, et al. (dir.), Clermont-Ferrand, Presses universitaires Blaise Pascal (Erga, 15), 2019.

Deux ans après la tenue, à Clermont-Ferrand, du dixième colloque de la section française de la société Rencesvals, la parution de ses actes, dans la belle collection Erga des Presses universitaires Blaise Pascal, permet d'apprécier les éclairages critiques récents portés sur la notion de sacré. Comme l'écrit justement Françoise Laurent, à qui l'on doit l'introduction du recueil (p. 9-16), « l'articulation de la chanson de geste et du sacré ne va $[\ldots]$ pas de soi ». C'est pourquoi, contrairement à leurs collègues des sciences humaines, peu de spécialistes de l'épopée médiévale ont proposé de travaux exclusivement consacrés à cette question (si l'on excepte l'importante thèse soutenue par Hubert Heckmann en 2010 « Le profane et le sacré dans les textes épiques médiévaux : 1100-1250»). On ne sera donc pas étonné de l'influence des réflexions, entre autres, d'Emile Durkheim, Mircea Eliade ou encore René Girard sur les différentes contributions de ce recueil.

Qu'est-ce que le sacré et en quoi intéresse-t-il la chanson de geste ? La notion demeure problématique, même si certains axes peuvent être dégagés. Rappelons d'abord, avec H. Heckmann, que le terme sacer dérive de sancire, «rendre inviolable», le sacré étant, selon E. Durkheim, frappé de tabou. En cela, la notion est ambiguë, puisqu'elle attire autant qu'elle ne repousse. Au Moyen Âge, le monde sacré est avant tout un monde « spatialisé » (représenté par le lieu " église »), ce qui ne l'empêche pas de s'incarner également dans des objets ou des personnes (justement « consacrés »), autant d'éléments qui peuvent avoir leur importance dans les chansons de geste. Mais le lien entre l'épopée et le sacré semble plus intime encore. H. Heckmann montre ainsi ce que la littérature épique profane pouvait avoir de commun avec l'hagiographie dans leurs célébrations d'une mémoire commune (ce qui distinguerait la chanson de geste du roman). Enfin, et ce dernier point mérite une attention toute particulière, le sacré dans la chanson de geste est bien souvent lié au statut royal. Comme l'écrit F. Laurent, «présente dans les chansons les plus anciennes, la sacralisation attachée à la personne du prince est une constante du genre épique médiévale » (p. 15). De fait, si la chanson de geste est bien idéologique (dans le sens où l'entendent Florence Goyet ou Dominique Boutet), alors le sacré participe pleinement de son esthétique.

La première partie de l'ouvrage ( «l'écriture du sacré : motifs; style et tradition textuelle », p. 19-134) regroupe des articles assez hétérogènes, représentatifs en cela d'une notion diffuse. Marianne Ailes interroge 
tout d'abord (p. 19-30) la place des reliques dans trois chansons qu'on a pu qualifier de « petit cycle des reliques », à savoir le Pèlerinage de Charlemagne, Fierabras et la Destruction de Rome. Si la quête de l'objet saint peut apparaître comme un simple motif diégétique (qu'on songe au Pèlerinage, où les reliques sont d'ailleurs l'un des rares objets à ne pas subir un traitement comique), la relique possède tout de même une force, à la manière du Saint Baume dans Fierabras (quand bien même celui-ci n'est guère respecté par Olivier après son utilisation). Une étude plus approfondie des reliques dans l'ensemble des chansons de geste reste à produire en comparaison avec ces trois œuvres. Jérôme Devard (p. 31-42) s'attarde quant à lui à la «sacralité du genus royal ». L'a. remarque que l'élection divine du roi de France, à la manière du Couronnement de Louis, n'est pas le modèle de la production épique. On trouve ainsi dans beaucoup de chansons de geste des « rituels d'intronisation faiblement christianisés » (remarquables notamment par l'absence de l'onction). En réalité, un certain nombre d'épopées plaident pour une royauté élective (Bertes as grans piés, Huon de Bordeaux, ...). En cela, nos textes se conforment à des réalités historiques (qu'on pense au « choix » de Pépin le Bref dans Garin le Loherenc). Cela ne signifie pas que la chanson de geste exempte la royauté de sacralité, mais celle-ci s'inscrit ailleurs, notamment dans le « charisme dynastique ». Si le roi épique n'est pas sacré, à proprement parler, il possède des « caractères magiques » qui ne sont pas sans rappeler « le pouvoir thaumaturgique des Capétiens » (p. 39). On appréciera au passage la recherche de lien entre la représentation de la royauté carolingienne et le contexte d'écriture capétien. Nadine Henrard (p. 43-59) nous rappelle quant à elle que « l'objet livre $\mathrm{a}[\ldots]$ des choses à nous dire » et s'attarde pour cela à l'étude de quelques manuscrits emblématiques associant des textes épiques à des textes sacrés. Cette démarche a le grand mérite d'éclairer la réception des chansons de geste par le biais de leurs copies. Ainsi, avec le manuscrit BnF nouv. acq. fr. 4232, qui associe deux unica, la Passion Didot et Daurel et Beton, deux œuvres dans lesquelles la thématique christique a une grande importance. En liant ces deux textes, « le copiste a en quelque sorte indiqué un parcours de lecture » estime l'auteure. À travers un échantillon limité, mais finement étudié (notamment du fameux codex L.II.14 de Turin), N. Henrard montre finalement que la frontière contemporaine entre genres religieux et profanes n'est pas des plus pertinentes. Il existe ainsi, écrit-elle, une certaine « insensibilité des acteurs de la diffusion [des textes] à la distinction entre les genres » (p. 59. N. Henrard renvoyant ici aux travaux d'Alberto Varvaro). Si, pour reprendre les termes de l'a., « ce chantier mérite plus ample exploration » cet article en ouvre la voie de belle manière. Revenant à la matière même des chansons de geste, Sandrine Legrand propose une étude de cas intéressante à travers la conversion difficile d'Hector de Salorie dans le Deuxième Cycle de la Croisade (p. 61-72). Alors que le motif de la conversion, personnelle ou collective, des Sarrasins est typique de la chanson de geste, les réticences d'Hector interrogent. Sans proposer de solution définitive, l'a. revient sur les différentes hypothèses de composition du texte. Si le cycle a été construit de manière linéaire, alors le personnage illustre un parcours dans la découverte de la foi chrétienne; si, en revanche, le cycle s'est construit par épisodes indépendants (comme les incohérences internes peuvent le suggérer), l'évolution du personnage doit être nuancée. On appréciera tout particulièrement ce type de démarche qui avoue modestement les limites du critique face aux traditions épiques. C'est dans le silence des chansons de geste que Valérie Méot-Bourquin (p. 73-89) part à la recherche du sacré. Après avoir rappelé la fonction du mutisme dans la religion et notamment dans la tradition monastique (règle de Saint Benoit par exemple), l'a. rappelle que la chanson de geste laisse peu de place au silence sacré. De fait, les héros épiques se recueillent peu, à l'exception notable de Guillaume dans le Couronnement de Louis. Changeant de perspective, V. Méot-Bourquin s'interroge ensuite sur la réception de la chanson de geste et sa participation au sacré. L'a. rappelle tout d'abord la forte présence de la religion dans nos textes, que ce soit par les personnages ou par les lieux. De plus, la violence constitutive de l'épopée n'est pas sans faire écho aux analyses de Rudolf Otho sur le sacré (l'exemple de l'incendie d'Origny dans Raoul de Cambrai est en cela particulièrement pertinent). Enfin, l'a. se demande si le sacré n'est pas à chercher dans le rythme et le style même des chansons de geste : « l'épopée est célébration et les procédés de la célébration sont ceux qui font naître le sentiment du sacré; grandissements, hyperboles, aphorismes, etc., tout concourt à opérer une distanciation, engendre 
un effet de majesté, créant une résonance générale d'élévation » (p. 87). Quoi de plus sacré que le nom de Dieu même, révéré et craint à la fois ? C'est aux différentes formes prises par le nom de la divinité que s'attache l'intéressante étude de Valérie Naudet. Deux formes semblent particulièrement privilégiées par les poètes épiques : la périphrase et la substantivation adjectivale. L'étude de la première est la plus intéressante : «à la fois figure de la ligne contournée et briseuse de la ligne grammaticale », elle « est pleinement partie prenante de l'esthétique de la chanson de geste » (p. 95). Une autre particularité de la dénomination de Dieu réside dans la possibilité d'accumulation, qui permet au poète de « cerner au mieux ce qui lui échappe » et participe d'une esthétique que l'a. qualifie à juste titre de « jubilatoire » (p. 95). L'article interroge ainsi tour à tour les différentes formules touchant à Dieu, en montrant in fine que l'amplification épique qui touche ce nom participe d'un art qui cherche à « célébrer plutôt qu'à évoquer » (p. 102). Ce sont les credo épiques qui intéressent quant à elle Muriel Ott (p. 103-120) dont l'étude s'attache à la tradition manuscrite de la Chevalerie Ogier. Après être revenue sur la complexité de cette tradition, l'a. se propose de comparer les six credo présents dans l'œuvre, selon qu'ils sont communs à l'ensemble des textes ( « credo certifié ») ou aux seuls remaniements. Il apparaît alors que ce motif a séduit les remanieurs de la chanson : ceux-ci ont en effet largement amplifiés les credo certifiés et surtout en ont ajouté un certain nombre à des « endroits soigneusement choisis » (p. 112). Ces credo récents n'ont cependant pas la fonction dramatique essentielle que revêtaient ceux de la première chanson (le credo des remaniements étant plus ornemental). L'article se conclut par une édition des épisodes étudiés dans l'ensemble de la tradition. La première partie de l'ouvrage se termine quant à elle par une réflexion que son auteur, Jan Zivcak, reconnaît lui-même comme polémique. Il s'agit d'une relecture alternative de la célèbre scène du sacre dans le Couronnement de Louis (p. 121-132). Selon l'a., cette scène reprendrait les trois étapes de la liturgie catholique : le sermon (qu'évoqueraient les différents discours de Charlemagne), la communion (la couronne remplaçant ici l'Eucharistie) et enfin l'offertoire. C'est ce dernier point qui pose difficulté, car elle suppose de lire la mort d'Ernaïs comme une forme de sacrifice. L'a. s'appuie ici sur les travaux de Mircea Eliade qui proposait l'existence de « sacrifice de construction » par le biais de la mort d'un ennemi « portant atteinte à la sacralité du Cosmos » (p. 126). Bien justifiée, cette théorie demeure hypothétique selon les mots mêmes de l'a. Elle montre cependant tout l'intérêt d'une approche pluridisciplinaire des chansons de geste, d'autant plus que la fin de l'article, consacrée à une relecture du lieu «mostier » selon les critères de M. Eliade paraît tout à fait pertinente : l'église dans lequel se passe le couronnement de Louis est un lieu sacré en ce qu'il s'oppose au profane, qu'il est civilisé et bien gardé, qu'il est identifié comme le centre du monde et qu'il est lié au sacrifice d'un être dangereux.

La deuxième partie de l'ouvrage porte de manière plus précise sur les " paradoxes du sacré » (p. 133-213) et s'ouvre sur les réflexions de Bernard Guidot quant à la Chanson d'Antioche (p. 135-160). La violence peut-elle être sacrée? Cette question, qui renvoie une fois encore aux travaux de M. Eliade, l'a. se la pose pour un texte qui associe dans le discours même de ses personnages la guerre et le sacré. Dans cette chanson de geste qui laisse une place importante au merveilleux chrétien, l'accent est mis surtout sur le bonheur qui résulte de l'alliance avec la divinité. Comme l'écrit B. Guidot : « le sacré des actes religieux procure une joie immense » (p. 140). Dorothea Kullmann revient quant à elle, dans un bel article, sur l'évolution du sentiment religieux tel qu'il transparaît dans le remaniement de Renaut de Montauban (p. 151-162). Le rapprochement que fait l'a. entre le glissement des personnages vers la sainteté et le contexte d'écriture du XIV ${ }^{\mathrm{e}} \mathrm{s}$. (notamment de l'émergence de la Dévotion moderne fondée par Gérard Grote) paraît intéressant. L'étude détaillée de l'épisode d'Angorie, durant lequel Renaut va devenir un « vassal exemplaire » (p. 160) est en tout cas parfaitement convaincante. Sous le titre provocateur de « Sacré Mahomet...! Traitements et maltraitance du divin dans Elie de Saint-Gilles » (p. 163-178) Beate Langenbrunch montre qu'avec cette chanson on « s'est sacrément éloigné de l'esprit de croisade pur et idéaliste » (p. 164; les italiques sont dans le texte). L'a. suspecte en réalité l'esprit chrétien de manquer de profondeur dans cette œuvre, qui, par exemple, fait étrangement peu référence aux saints. L'étude des prières est éclairante à ce sujet, et on appréciera la pertinence de la surprenante comparaison entre la matérialité des prières d'Elie et la 
chanson de Janis Joplin « Oh Lord, would you buy me a Mercerdes Benz » (p. 173-174). C'est finalement, " paradoxes du sacré », Rosamonde, la païenne en voie de conversion, qui semble faire preuve d'un vrai sentiment religieux. Chloé Lelong (p. 179-190) revient pour sa part sur « l'infléchissement certain de l'image du souverain » (p. 179) dans les épopées francoitaliennes. Si le phénomène est connu en France dès le $\mathrm{XII}^{\mathrm{e}} \mathrm{s}$., il est encore accru dans les textes italiens. Ainsi, dans la Prise de Pampelune, Charlemagne n'apparaît pas forcément comme un roi exemplaire et multiplie les torts. On notera cependant, comme l'écrit l'a., que si Charlemagne peut être dépeint comme un " plaisantin », « la désacralisation de sa personne ne l'empêche pas de rayonner » (p. 190). Ce sont les « dieux incapables » des Sarrasins qui intéressent Pierre Levron. Sa communication (p. 191-202) s'attarde tout d'abord sur le " polythéisme confus » que dépeignent les chansons de geste (avec un renvoi utile aux travaux de Paul Bancourt). L'étude de différentes scènes de désespoir des païens face à l'incapacité de leurs dieux à leur donner la victoire montre la proximité du genre épique avec la littérature polémique de l'époque contre l'Islam. Ce deuxième temps de l'ouvrage se termine par une belle contribution d'Elena Podetti sur quelques « aspects du sacré dans la Chanson d'Ydes et Olive ", texte que connaît bien l'a. pour l'avoir édité dans le cadre de son doctorat. Après avoir rappelé ce que cette chanson de geste doit aux motifs folkloriques de Peau d'Ane et de La fille aux mains coupées, E. Podetti montre que ces derniers se retrouvent également dans la littérature hagiographique (on appréciera au passage le soin pris dans l'élaboration de la riche bibliographie infrapaginale). Une distinction cependant s'impose : alors que les textes religieux évoquent la possibilité d'une pilosité féminine dissimulant la nature des personnages, ils ne vont pas jusqu'au changement de sexe imaginé par le poète épique. L'a. établit clairement qu'Yde et Olive s'inscrit dans une réflexion médiévale autour de deux tabous : l'inceste et l'homosexualité (rappelant au passage que « jusqu' au XIII ${ }^{\mathrm{e}}$ s., l'Église ne cesse pas de légiférer sur les interdits sexuels et matrimoniaux », p. 211). Il est intéressant de noter que ce qui semble véritablement poser problème avec l'homosexualité dans ce texte est l'absence de descendance qu'elle implique.

La troisième partie de l'ouvrage porte sur le lien ambigu entre « le sacré et la violence » (p. 215-282), reprenant notamment les thèses (non discutées ici) de René Girard sur la violence mimétique. C'est Emma Bahillo Sphonix-Rust qui ouvre cette partie avec une présentation intéressante et contrastée des personnages de Floripas dans Fierabras et de Berte dans la chanson éponyme (p. 217-225). Si la première héroïne fait sienne la violence, c'est qu'elle défend une cause sacrée en protégeant des reliques. Quant à la seconde, c'est en étant victime de la violence, qu'elle accepte avec résignation, qu'elle accédera au sacré. Philip E. Bennett s'intéresse quant à lui à la violence des croisades, notamment dans La Chanson d'Aiquin (p. 227-237). Après avoir rappelé que l'état fragmentaire du texte rendait sa lecture difficile, l'a. s'interroge sur le véritable héros du texte : s'agit-il vraiment de Charlemagne, ou bien d'Ysoré, l'archevêque de Dol (hypothèse de Nicolas Lenoir à qui est dédié cet article)? Il apparaît en tout cas que la campagne militaire menée par les Français en Bretagne n'est pas conforme au modèle classique de la chanson de geste. L'exemple bien étudié de la première grande bataille de Quidalet est remarquable en cela avec la mise en place d'une « bataille de trois jours », " calquée sur les tournois de trois jours des romans arthuriens » (p. 232). L'a. termine en s'interrogeant sur l'importance du thème du pèlerinage dans l'œuvre à travers les différents arrêts de l'empereur, qui réaffirme ainsi son « rôle spirituel » (p. 237). Marie-Madeleine Castellani (p. 239-248) revient dans une riche communication sur un épisode précis du Récit en vers de la première croisade d'après Baudri de Bourgueil (que l'a. propose fort heureusement d'abréger $R P C B B$ ). L'affrontement de Saint Pierre et de Simon le magicien, inconnu de l'original latin, peut intriguer, d'autant qu'il est évoqué à trois reprises dans le texte. M.-M. Castellani revient sur les sources apocryphes de cet épisode avant d'établir une chronologie possible des événements qui auraient vu Pierre chasser Simon d'Antioche avant de le vaincre à Rome. Surtout, l'a. montre que «l'intention de l'a. du RPCBB est apologétique »: en se référant à Saint Pierre, l'a. vient justifier l'action des croisés à Antioche. Le grand mérite de l'article d'Alain Corbellari (p. 249-257) est de mettre en lumière un texte à la " maigre fortune critique » : La vengeance Notre Seigneur. L'a. revient sur les caractéristiques de ce texte, qui est un des «plus sinistres que l'on puisse lire » (p. 251), et notamment sur la multiplication des scènes insoutenables (femme 
contrainte de manger son fils, etc.). On rejoindra l'a. qui conclut qu'en s'intéressant aux débuts du christianisme, cette chanson ne pouvait être que « surclassée par des exploits d'un christianisme enfin dénué de toute ambiguïté » (p. 257). Dans « Garin le Loherenc à l'épreuve de La violence et le sacré de René Girard» (p. 259-271), Philippe Haugeard essaie de lire la première pièce du cycle des Loherains à la lumière de l'hypothèse du désir mimétique. Selon l'anthropologue R. Girard, l'opposition mimétique de deux forces plonge la communauté dans une crise qui la menace d'autodestruction. La solution résiderait alors dans le recours à la violence contre un seul être jugé responsable de la violence de tous, la « victime émissaire ». Selon P. Haugeard, il y aurait ainsi un désir mimétique de Fromont concernant Garin et les morts des deux premiers Lorrains peuvent être considérées, dans cette hypothèse, comme des sacrifices de victimes expiatoires. L'ouvrage se termine par un autre article en lien avec les théories girardiennes, cette fois appliquées à la " petite geste de Blaye » (p. 273-282). Bernard Ribémont évoque tout d'abord une sorte d'incohérence dans les textes, puisque Jourdain semble doué d'une prescience surnaturelle quand il fait référence au jugement d'Hardré qui a lieu dans l'autre chanson du cycle ; incohérence qui n'en serait pas forcément une, la chanson de Jourdain pouvant en réalité proposer « la solution d'une forme de crise sacrificielle » (p. 275). De fait, selon l'a., la conclusion d'Ami et Amile, avec le « sacrifice" d'Hardré ne serait pas suffisante pour résoudre pleinement la crise de la communauté; c'est pourquoi la mort de Fromont, dont la faute, contrairement à celle d'Hardré, est " exemplaire et dénuée d'ambiguité » (p. 282), vient clore de manière définitive le cycle. Les réflexions de l'a. sur la construction du cycle peuvent ouvrir à de nouvelles lectures éventuellement transposables à d'autres cycles (nous pensons par exemple à la Geste des Loherains).

Si la chanson de geste cherche bien à « célébrer avec solennité, dans un langage rituel, la liturgie de l'héroïsme chevaleresque ", pour reprendre le mot fameux de Pierre le Gentil ( À Propos de la Chanson de Roland et la tradition épique des Francs de Ramon Menendez Pidal », Cahiers de civilisation médiévale, 19, 1962, p. 323-333, DOI : https://doi. org/10.3406/ccmed.1962.1237, ici p. 332), les différentes études de ce recueil permettent d'en mieux établir les correspondances avec la foi qui les anime, mais aussi ses paradoxes. Un certain nombre d'hypothèses formulées dans ce cadre méritent assurément un complément d'enquête, notamment en ce qui concerne le contexte d'écriture des chansons de geste. Il apparait en tout cas assez clairement que le sacré médiéval, dont la distinction avec le profane ou le laïc n'a pas la netteté de nos vues contemporaines, par son lien avec l'idéologie royale, devrait avoir une place de choix dans nos réflexions sur les textes épiques.

Gauthier GRÜBER

EA 4343 - Calhiste/UVHC 